\title{
ANALYSIS OF SPECTRUM SENSING TECHNIQUES FOR DETECTION OF DVB- T SIGNALS IN GAUSSIAN AND FADING CHANNELS
}

\author{
Ireyuwa Igbinosa ${ }^{1}$ Olutayo Oyerinde ${ }^{2}$ and Stanley Mneney ${ }^{1}$ \\ School of Electrical, Electronic and Computer Engineering, ${ }^{1}$ \\ University of KwaZulu-Natal, Durban, 4041, South Africa. \\ Tel: +27744200748 \\ School of Electrical and Information Engineering, ${ }^{2}$ \\ University of the Witwatersrand, Johannesburg, 2050, South Africa. \\ yvwa123@gmail. $\mathrm{com}^{1}$; olutayo.oyerinde@wits.ac.za ${ }^{2}$; \\ mneneys dukzn.ac. za ${ }^{1}$
}

\begin{abstract}
Spectrum sensing is an essential concept in cognitive radio. It exploits the inefficient utilization of radio frequency spectrum without causing destructive interference to the licensed users. In this paper we considered spectrum sensing of Digital Video Broadcast Terrestrial (DVB-T) signal in different scenario. We compared various spectrum sensing algorithms that make use of the second order statistics; the energy detector was also included for comparison. The results show that it is possible to obtain good detection performance by exploiting the correlation method.
\end{abstract}

\section{KEYWORDS}

Spectrum sensing, DVB-T, OFDM, Cognitive Radio

\section{INTRODUCTION}

Spectrum sensing can be said to be the process of performing measurement on a part of a spectrum and thereby forming a decision based upon measured data [1]. Spectrum sensing is an essential operational block of the cognitive radio which consists of spectrum sensing, management and spectrum mobility. Measurement of spectrum has shown unused spectrum resources in frequency, time and space [2]. The frequency bands of the wireless communication are currently not efficiently used. This is due to the strict frequency allocation policy. The issue of spectrum utilization brought about the cognitive radio concept. This concept has proven itself as a promising technique to improve spectrum utilization by exploiting the spectrum holes. However, the introduction of cognitive Radio in an existing network increases interference. Hence the impact on the primary network must be kept as low as possible. Therefore the

David C. Wyld et al. (Eds) : SAI, CDKP, ICAITA, NeCoM, SEAS, CMCA, ASUC, Signal - 2014 pp. 343-353, 2014. C CS \& IT-CSCP 2014

DOI : $10.5121 /$ csit.2014.41131 
secondary users must sense the spectrum and detect whether the primary user is occupied. For this to be achieved, the secondary users should be able to detect very weak primary user signals [4].

A notice of proposed the rulemaking (NPRM) issued by the U.S. federal communication commission (FCC) in 2004 indicated that the unutilized TV Channels in both very high frequency (VHF) and ultra high frequency (UHF) bands could be used for fixed broadband access [5]. This factor increased the interest within the research community to develop a standard for wireless regional area network (WRAN) system operating on a TV white space making use of cognitive Radio techniques [6]. According to the IEEE 802.22 standard a secondary user should be able to detect a primary user of DVB-T signal with the probability of detection of at least 0.9 and the probability of false alarm of not more than 0.1 , at $-22.2 \mathrm{~dB}$ SNR [7-8]. The cognitive functionality of this standard is channel sensing, channel classification and maintenance of channel information. A description of all the functionalities of an 802.22 WRAN is given in [7].

In this research, we evaluated various detection algorithms for detecting primary DVB-T signals in different scenarios. We considered a number of feature detectors and compared them with energy detectors.

The rest of this paper is organized as follows, section II, show characteristics of DVB-T signals, section III, problem formulation and section IV shows the various detection algorithms, section $\mathrm{V}$ shows the simulation results and finally section VI concludes this paper.

\section{CHARACTERISTICS OF DVB-T SIGNALS}

From the draft of the European Telecommunication Standard Institute (ETSI), the characteristics of the physical layer were discussed in detailed [9]. But in the scope of this work, we are going to summarize our main area of interest which is the channel coding and modulation. The system input stream which is organized in fixed length packets of the digitized multiplexed MPEG-2 signal that carries the payload data is divided in lengths of 188bytes [9]. The major modulation constellation used are QPSK, 16 QAM, 64 QAM, non-uniform 16 QAM, or non-uniform 64 QAM. Details can be found in section 4.3.5 in [9]. The modulated signals are determined by three main parameters which include; bandwidth, mode and length of the cyclic prefix. The bandwidth usually takes one of the following values $5 \mathrm{MHz}, 6 \mathrm{MHz}, 7 \mathrm{MHz}$ or $8 \mathrm{MHz}$. The number of subcarriers that are used in the OFDM modulation are determined by the mode parameters which can take values of $2 \mathrm{k}$ or $8 \mathrm{k}$ mode which is 2048 or 8192 respectively. The cyclic prefix is used in order to avoid inter symbol interference (ISI) in wireless transmission. The length of the cyclic prefix is determined in terms of fractions of the duration of OFDM symbol part and assigns the values $1 / 4,1 / 8,1 / 16$ and 1/32. A sequence of 68 consecutive OFDM symbols constitutes a frame and four of such frames are gathered to form a super frame. The continuous DVB-T signal in time domain can be defined as:

$$
s(t)=e^{j 2 \pi f c^{t}} \sum_{m=0}^{\infty} \sum_{i=0}^{67} \sum_{k=0}^{k \max } c_{m, i, k} \varphi_{m, i, k}(t)
$$




\section{SYSTEM MODEL}

It is assumed that the DVB-T Signal mode is known; hence the parameter of the DVB-T signal that we want to detect is known. Let $\mathrm{x}(\mathrm{t})$ be the received continuous baseband signal, then the time received sequence becomes:

$$
s[k]=x\left(k T_{e}\right), k=0,1, \ldots \ldots M-1,
$$

where $\mathrm{M}$ is the total number of samples. The difficulty of spectrum sensing is to decide whether there is transmitted signal or not. Hence we have to be able to discriminate between the following hypotheses:

The $\mathrm{H}_{0}$ and $\mathrm{H}_{1}$ correspond to either the absence or presence of DVB-T signal. The sequence $\mathrm{s}[\mathrm{k}]$ is the sampled version of the signal $\mathrm{s}(\mathrm{t})$ defined in equation (1). Where $f_{c}=0$ and sampling rate is $1 / T_{e}$. The amplification factor hi and the delays ki describe the multipath fading environment. The model assumes that the signal propagates through multipath fading environment. The noise $n(k)$ is assumed to be complex white zero-mean Gaussian with variance ${ }^{\sigma_{n}^{2}}$. The decision on the presence or absence of DVB-T signal is based on a test statistics $\gamma_{\text {which }}$ is a result of the received sequence $\{\mathrm{x} \mid \mathrm{k}\}_{k=0}^{M-1}$, i.e

$$
\gamma=f(x[0], x[1], \ldots \ldots \ldots \ldots x[M-1])
$$

In order to decide upon a hypothesis we compare $\gamma$ to a threshold $(\mu)$ and decide whether the signal is present. The probability of false alarm (Pfa) and the probability of detection are defined as:-

$$
\begin{aligned}
& \text { Pfa }=\operatorname{Pr}\left\{\gamma>\mu \mid H_{0} \text { is true }\right\} \\
& \text { and } \quad \operatorname{Pd}=\operatorname{Pr}\left\{\gamma>\mu \mid H_{1} \text { is true }\right\}
\end{aligned}
$$

These are the two main detector performance indicators. The test statistic can be chosen in different ways in the rest of the paper we would show some of the choices.

\section{SPECTRUM SENSING ALGORITHMS}

This section presents the various algorithms that were considered for the detection of the DVB-T signals.

\subsection{Energy Detection}

Energy detection is an optimal way to detect primary signals when prior information of the primary signal is unknown to the secondary user. It measures the energy of the received signal waveform over a specified observation time [10-11] and compares it to a predefined threshold. The test statistic for energy detector is: 


$$
\gamma_{E D} \triangleq \sum_{k=0}^{M-1} x[k] x^{*}[k]
$$

The performance of the energy detector is well known and can be written in closed form. The probability of false alarm $\mathrm{P}_{\mathrm{FA}}$ is then given as

$$
P_{F A} \triangleq P_{r}\left(\gamma_{E D}>\mu_{E D} \mid H_{0}\right)=1-F x_{2 M}^{2}\left(\frac{2 \mu_{E D}}{\sigma_{n}^{2}}\right)
$$

Where $F x_{2 M}^{2}($.$) is the cumulative distribution function of \mathrm{ax}^{2}$ - distributed random variable with $2 \mathrm{M}$ degrees of freedom. Therefore, given a false alarm. Then we can derive the threshold $\mu_{E D}$ from the following equation :-

$$
\mu_{E D}=F_{x_{2 M}^{2}}^{-1}\left(1-P_{F A}\right) \frac{\sigma_{n}^{2}}{2}
$$

The probability of detection is then defined as:-

$$
P_{r}\left(\gamma_{E D}>\mu_{E D} \mid H_{1}\right)=1-F x_{2 M}^{2}\left(\frac{2 \mu_{E D}}{\sigma_{n}^{2}+\sigma_{s}^{2}}\right),
$$

where $\sigma_{s}^{2}$ the average is received signal power. From equation 5 it shows that the energy detector requires the noise power $\sigma_{n}^{2}$ to be known, else the detector would perform poorly. However, it is a known fact that the energy detector deteriorates when noise power estimate is imperfect [12].

\section{FEATURE DETECTORS BASED ON SECOND ORDER STATISTICS}

The term feature detector is mainly used in the context of spectrum sensing and usually refers to the exploitation of known statistical properties of the signal [13]. The features of the transmit signals are results of the redundancy added by coding of the modulation and formatting schemes used at the transmitter end. For example OFDM modulation adds a cyclic prefix that manifest itself through a linear relationship between the transmitted samples. It is also known that most communication system multiplex known pilot symbol into transmitted data stream or better still superimpose pilot symbol on the transmitted signals and by so doing it results into very destructive signal feature.

In this section we would show the simulation results of some state of the art detectors that exploit signal features suitable for spectrum sensing application in cognitive radio. The methods proposed here are recent advances in spectrum sensing and there are still ongoing researches in this area.

\section{A. Autocorrelation Based Detection}

The autocorrelation detector is a simple and computationally efficient spectrum sensing scheme for OFDM based primary user signal using its autocorrelation coefficient. OFDM signals have a very explicit correlation structure imposed by the insertion of a cyclic prefix (CP) at the transmitter [14]. This method exploits the correlation of an OFDM signal using knowledge of the 
Td. The method proposed in [14], uses the empirical mean of the sample value product $\mathrm{r}[\mathrm{k}]$, normalized by the received power as test statistic. The theoretical approximation of the values of the threshold and probability of detection are given in equation 20-21 [14]. The noise variance does not need to be known to make a decision. The test statistics is defined in [14] is given as-

$$
\gamma_{A C D}=\frac{\frac{1}{M-T_{d}} \sum_{k=0}^{M-T_{d}-1} \operatorname{Re}(r[k])}{\frac{1}{M \sum_{k=0}^{M-1}|x[k]|^{2}}}
$$

\section{B. Detection Based on Cyclic Prefix Sliding Window Correlation}

To protect the DVB-T signal against ISI, a CP which copies the last part of the symbol is added at the beginning of each OFDM symbol. The duration of the CP could be 1/4,1/8,1/16 or 1/32 of the original symbol duration. The detector of [15] uses a sliding window that sums $T_{c}$ which is the consecutive samples. The approach of taking the sum of the real part was proposed in [16] the test statistics is given as;-

$$
\gamma_{S W}=\max _{\theta} R_{e}\left(\sum_{k=0}^{\theta+T_{c}-1} R[k]\right)
$$

\section{Detection based on second order statistics}

The repetition of data in the CP causes a structure to an OFDM signal. This structure can be exploited for detection. The detector proposed in [13] is assumed to know the size of the OFDM symbol that is $T_{c}$ and $T_{d}$ and exploits the structure obtained by the CP, where $S_{\theta}$ indicates the $T_{c}$ consecutive correlated samples. Detailed equation is given in [13]. Contrary to the Energy detector, this does not require any prior knowledge of the noise power. The test statistic for this proposed detector is given as:-

$$
\gamma_{2 n d}=\max _{\theta}\left(\sum_{k \in s_{\theta}} \operatorname{Re}(R[k])\right)^{2}
$$

\section{SIMULATIONS AND RESULTS}

In this section, we present the simulated results of the spectrum sensing algorithms described in previous sections showing their comparisons. Five scenarios were considered, the DVB-T signal model is the same for all scenario. The following setting was used for all scenarios:

- DVB-T signal model: $2 \mathrm{k}$

- Cyclic prefix: $\mathrm{Tc} / \mathrm{Td}=1 / 4$

- Probability of false Alarm: 0.05 
- DVB-T bandwidth: $8 \mathrm{MHz}$

- Number of realization for simulated Pd: 1000

\begin{tabular}{|c|c|}
\hline Abbrevation & Detector \\
\hline $2^{\text {nd }}$ Order & Detection based on second order statistics \\
\hline ACD & Autocorrelation based detector \\
\hline SW & $\begin{array}{c}\text { Detection based on cyclic prefix sliding } \\
\text { window }\end{array}$ \\
\hline ED & Energy Based detector \\
\hline
\end{tabular}

Table 1. Detectors Simulated

\section{Scenario 1}

In this scenario AWGN channel was considered with the sensing time of $10 \mathrm{~ms}$ and Pfa of 0.05 using 1000 simulations. The results are shown in figure 1

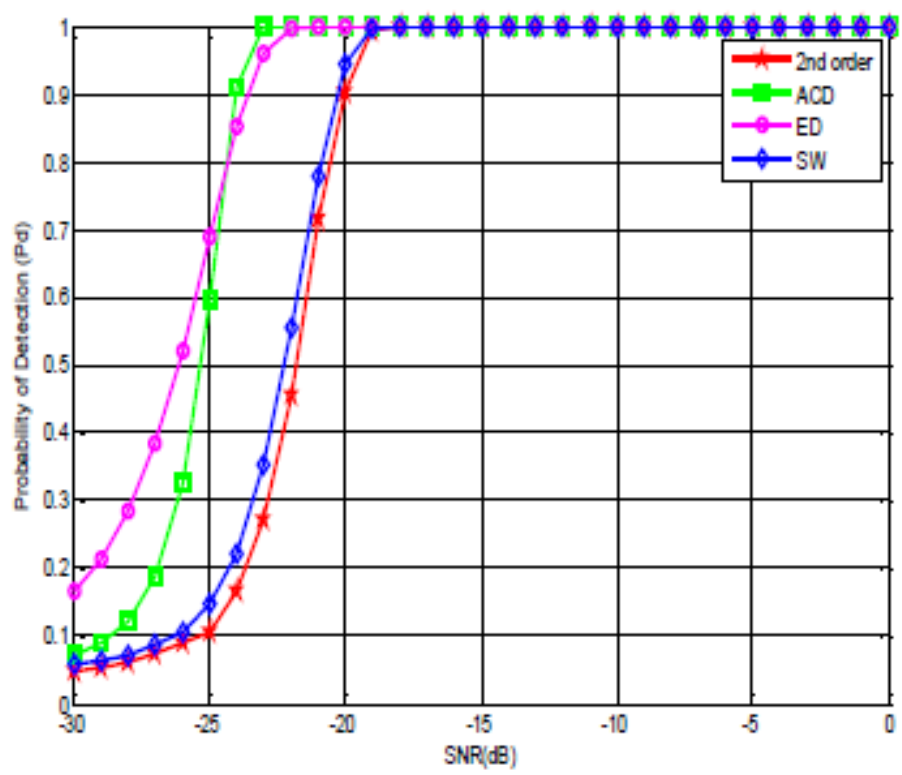

Fig.1. Probability of detection for AWGN channel sensing time $10 \mathrm{~ms}$

\section{Scenario 2}

In this scenario the AWGN channel was considered using the sensing of 50ms and Pfa of 0.05 using 1000 simulation 


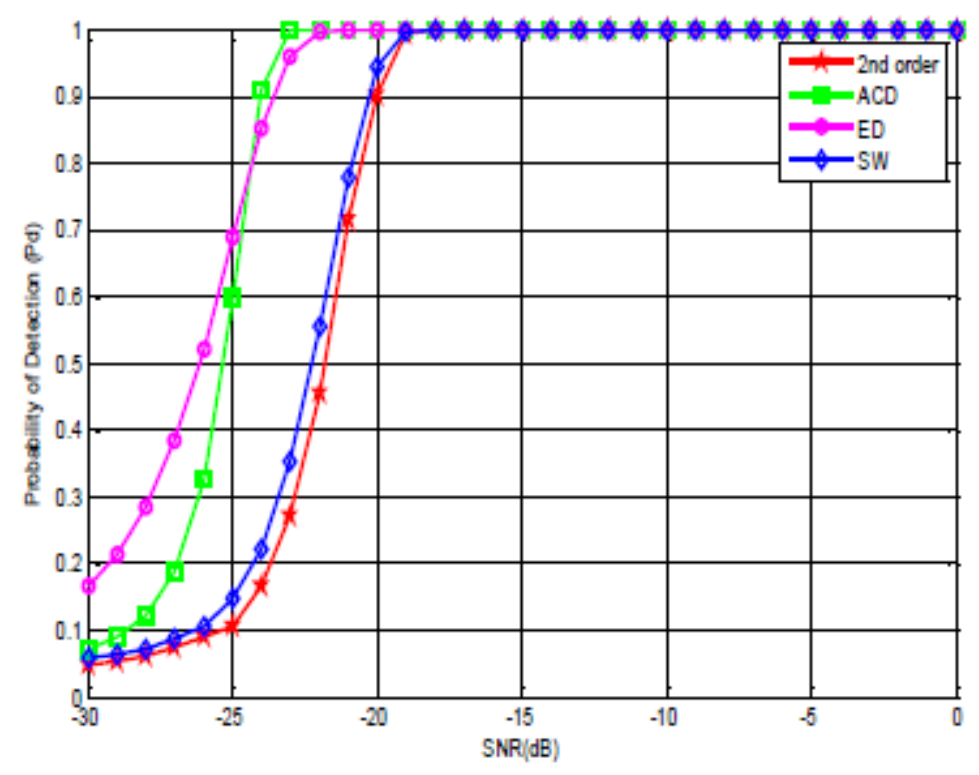

Fig.2. Probability of detection for AWGN channel sensing time 50ms

\section{Scenario 3}

In this case a Rayleigh flat fading channel was considered using the built in Matlab fading generators with a sensing time of $10 \mathrm{~ms}$ and Pfa of 0.05 using 1000 simulation result are shown in figure 3

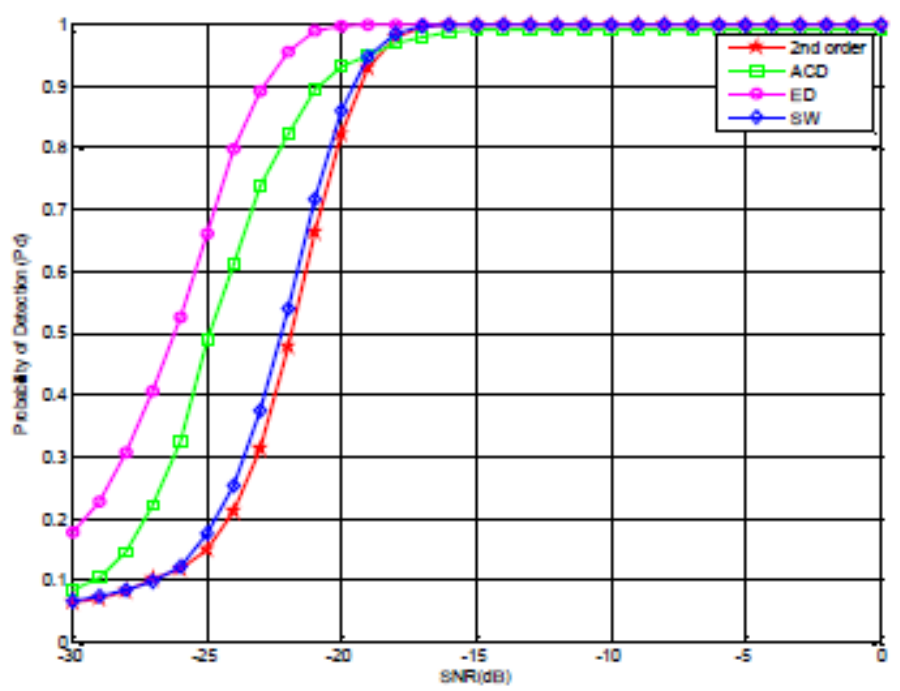

Fig.3. Probability of detection for Rayleigh flat fading $10 \mathrm{~ms}$ sensing time 


\section{Scenario 4}

In this case a Rayleigh flat fading channel was considered using the built in Matlab fading generators with a sensing time of 50ms Pfa of 0.05 using 1000 simulation result are shown in figure 4.

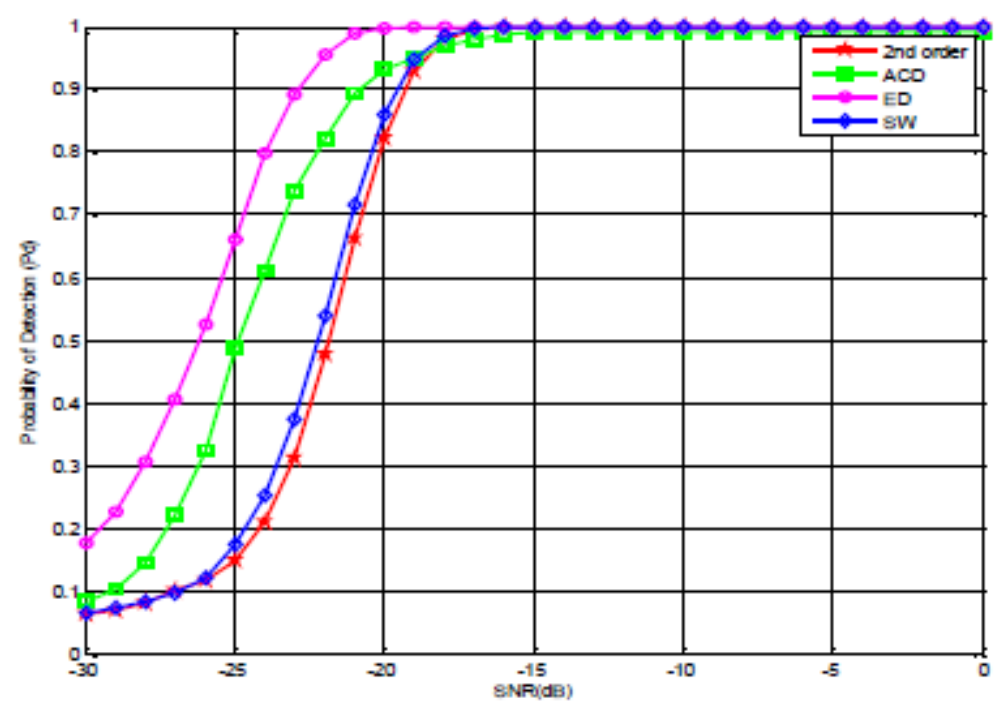

Fig.4. Probability of detection for Rayleigh flat fading 50ms sensing time

\section{Scenario 5}

In this section a Rayleigh flat fading channel with shadowing was considered with $10 \mathrm{~ms}$ sensing time. The standard deviation of the $\log -$ normal shadowing is $10 \mathrm{~dB}$. The results are shown in figure 5

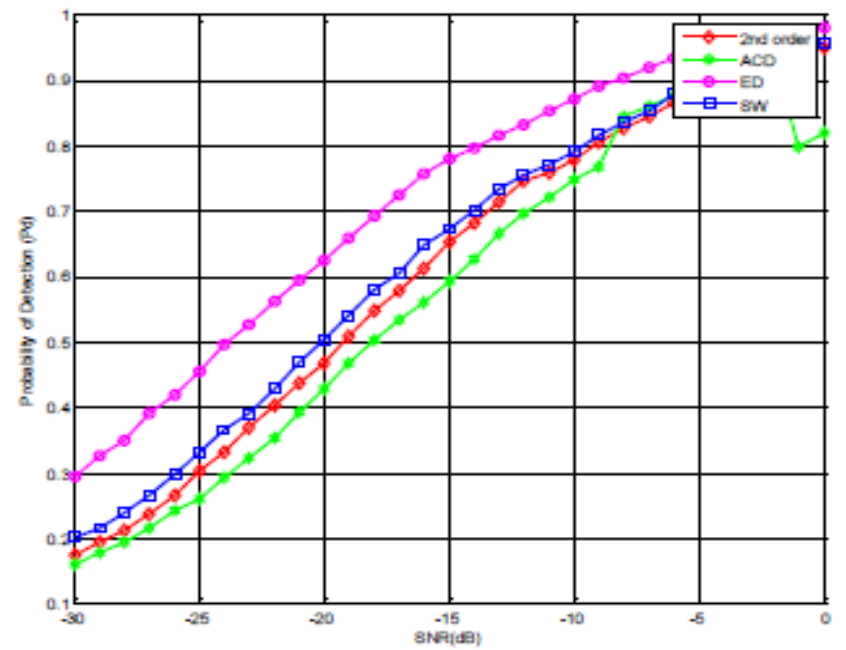

Fig.5. Probability of detection for Rayleigh flat fading channel with shadowing 10ms sensing time 


\section{Scenario 6}

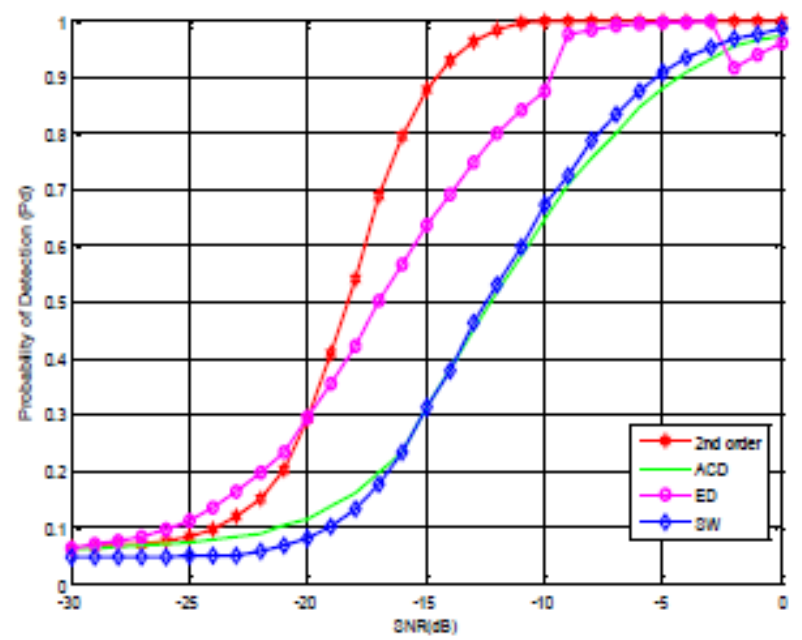

Fig.6. Probability of detection for Rayleigh multipath fading with $10 \mathrm{~ms}$ sensing time

In this scenario the Rayleigh multipath fading channel was considered using the extended vehicular model using the sensing $10 \mathrm{~ms}$. The simulated channel model was done by the built in functions in MATLAB for Rayleigh fading environment.

The presented algorithms do not rely on any information about the structure of the waveform; the only assumption is the knowledge of the CP and the total duration of OFDM symbol of the feature detectors while the energy detector does not require prior knowledge of the signal. Though the energy detector tends to show better performance among the others, its major drawback is that it assumes perfect knowledge of the noise floor level. Therefore, if the noise power is known the energy detector works excellently well. The performance of the energy detector is drastically affected id the noise power knowledge is erroneous. In practical scenario the noise power is never perfectly known hence estimated. Also if there is interference i.e. a signal from another secondary user, the feature detectors would be able to distinguish between the primary signal and the interfering signal whereas the energy detector would not be able to make the detection. In the case were the knowledge of the noise power is completely unknown only the second order statistics and the autocorrelation detectors would be able to work effectively. From observation of the graphs it can be seen that the second order statistics out performs the autocorrelation based detector in all scenario in the region of $\mathrm{P}_{\mathrm{d}} \geq 0.15$.

When we observe the result of the Rayleigh multipath fading channel, we can see that the channel does not much influence on the performance of the presented detectors because a frequency selective channel, exploits the inherent correlation of the OFDM signal obtained by repetition of data caused by the cyclic prefix is then exploited for detection.

\section{CONCLUSION}

In this paper we considered various sensing algorithms which uses the second order statistics for the detection of the DVB-T signal and compared the result to the energy detector. The second order based statistics method performed well with less information and they would make good 
candidates for implementation in cognitive Radio spectrum sensor. In this work we did not consider the carrier frequency offset (CF0) because such imperfect knowledge would decrease the correlation structure of the OFDM signal. In future we would make comparison of spectrum sensing algorithms under such conditions.

\section{ACKNOLEDGEMENT}

The Author would like to appreciate the centre for postgraduate funding (CEPS) for their funding and support towards this work.

\section{REFERENCES}

[1] End to End Efficiency (E3) white paperhttp:// www.ict-e3.eu, 2004.

[2] FCC,"Spectrum policy task force report", Technical report 02-135, Federal communication commission, Nov.2005, available http://transition.fcc.gov/sptf/files/SEWGfinalReport_1.pdf.

[3] S.Haykin, "Cognitive radio: brain-empowered wireless communication," IEEE Journal on selected areas in communication, vol.23, no.2, pp.201-220, Feb. 2005.

[4] A. Sahai, N.Hoven and R.Tandra, "Some fundamental limits on cognitive Radio", in Allerton Conference on communication control and computing, Oct.2004, pp.1662-1671.

[5] U.S. FCC, ET Docket 04-186, "Notice of proposed rulemaking in the matter of unlicensed operation in TV Broadcast Bands", May 2004.

[6] G.ko,A.A.Franklin, S-J You, J-S Pak, M-Y Song and C-J Kim, "Channel management in IEEE 802.22 WRAN systems", IEEE Communication magazine, Sep. 2010.

[7] C.Stevenson et al., "IEEE 802.22: The first Cognitive Radio wireless regional area network standard", IEEE Communication magazine, vol.47, no.1, pp.130-138, Jan.2009.

[8] S.Shellhammer and G.chouinard, "Spectrum Sensing Requirements Summary", IEEE Standard, 8022.22-06/0089r4. June 2006.

[9] ETSI EN300 744 VI.6.1 (2009-01), 'Digital Video Broadcasting (DVB), framing structure, channel coding and modulation for digital terrestrial television.” Technical Report, ETSI, 2009.

[10] H. Urkowitz, "Energy detection of Unknown deterministic signals", Proceedings of IEEE, vol.55, no.4, pp.523-531, Apr. 1967.

[11] M. Hoyhtya, A. Hekkala, M.Katz and A. Mammela, "Spectrum Awareness: Techniques and challenges for active spectrum sensing", Cognitive wireless networks, pp. 353-372, 2007.

[12] R. Tandra and A.Sahai, "Fundamental limits and detection in low SNR under noise uncertainty", in IEEE international conference on wireless networks communication and mobile computing, June 1316, 2005, vol.1, pp.464-469.

[13] E. Axell, "Spectrum Sensing Algorithm Based on Second Order Statistics" Ph.D. Thesis, Division of communication system, Department of Electrical Engineering, linkoping University, 2012.

[14] S.Chaudhari, V.koivunen and H.V.Poor "Autocorrelation based decentralized sequential detection of OFDM signals in cognitive radio", IEEE Transaction on signals processing, vol.57, no.7, pp.26902200 , July 2009.

[15] Huawei Technologies and UESTC “Sensing Scheme for DVB-T” IEEE Standard 802.22-06/0127-1, July 2006.

[16] D. Danev, "On Signal Detection techniques for DVB-T Standard" Proceeding of the 4th International Symposium on communication control and regional Processing (CIP) 2010.

[17] H.L.Van Trees. "Detection, Estimation and Modulation Theory: Part 1, John Wiley and sons Inc. 1968. 


\section{AUTHORS}

Ireyuwa Eghosa Igbinosa received his Diploma in computer engineering from the University of Benin, in 2003, B.Eng. and M.Sc by Research in Manchester Metropolitan University in Manchester United kingdom in 2011 and currently working on a Ph.D. at the University of KwaZulu-Natal, Durban. His research interest include cognitive radio application, wireless system, Spectrum sensing and OFDM systems.

Olutayo Oyeyemi Oyerinde received the B.Sc. (Hons.) and the M.Sc. from Obafemi Awolowo University, Ile-Ife, Nigeria, in 2000 and 2004, respectively, and the Ph.D. degree from the School of Engineering, University of KwaZulu-Natal (UKZN), Durban, South Africa, in 2010. He is currently a Telecommunications lecturer in the School of Electrical and Information Engineering, University of the Witwatersrand, South Africa. His research interests includes multiple antenna systems, orthogonal frequency division multiplexing system and channels estimation, and signal processing techniques.

Stanley Henry Mneney received the B.Sc. (Hons.) Eng. degree from the University of Science and Technology, Kumasi, Ghana, in 1976 and the M.A.Sc.from the University of Toronto, Toronto, Ontario, Canada, in 1979. In a Nuffic funded project by the Netherlands government he embarked on a sandwich Ph.D programme between the Eindhoven University of Technology, Eindhoven, Netherlands and the University of Dares Salaam, Dares Salaam, Tanzania, the latter awarding the degree in 1988. He is presently a Professor of Telecommunication and Signal Processing at the University of KwaZulu-Natal, Durban, South Africa. His research interests include theory and performance of telecommunication systems, low cost rural telecommunications services and networks, channel modelling and digital signal processing applications. 